\title{
The Effect of Particle Size on the Interpretation of Pore Structure of Shale by $\mathbf{N}_{2}$ Adsorption
}

\author{
Chengfu Lyu $\mathbb{D},,^{1,2}$ Xinmao Zhou $\mathbb{D}^{3}$, Xuesong Lu $\mathbb{D}^{3,4}$ Ying Zhang $\mathbb{D}^{1,5}$ Chao Li $\mathbb{D}$, ${ }^{1,2}$ \\ Qianshan Zhou $\mathbb{1}^{1},{ }^{1}$ Zhaotong Sun $\mathbb{1}^{1,5}$ and Guojun Chen $\mathbb{1}^{1,2}$ \\ ${ }^{1}$ Northwest Institute of Eco-Environment and Resources, Chinese Academy of Sciences, Lanzhou 730000, China \\ ${ }^{2}$ Key Laboratory of Petroleum Resources, Lanzhou, Gansu Province 730000, China \\ ${ }^{3}$ Research Institute of Petroleum Exploration \& Development, PetroChina, Beijing 100083, China \\ ${ }^{4}$ Key Laboratory of Basin Structure and Hydrocarbon Accumulation, CNPC, Beijing 100083, China \\ ${ }^{5}$ University of Chinese Academy of Sciences, Beijing 100049, China
}

Correspondence should be addressed to Chengfu Lyu; chengfu_lyu@126.com

Received 27 August 2020; Revised 30 September 2020; Accepted 13 April 2021; Published 24 April 2021

Academic Editor: Mohamed A. El-Beltagy

Copyright (c) 2021 Chengfu Lyu et al. This is an open access article distributed under the Creative Commons Attribution License, which permits unrestricted use, distribution, and reproduction in any medium, provided the original work is properly cited.

\begin{abstract}
Gas adsorption experiments are becoming one of the most common methods to quantify and analyze the pore structures of shale samples in the petroleum industry. In this regard, particle size of the specimen plays an important role in the results that could ultimately affect the pore structure interpretation. Hence, in this study, five shale samples at different thermal maturity levels are picked, and all are crushed into different groups of particle sizes: less than 40 mesh $(<375 \mu \mathrm{m})$, less than $60 \mathrm{mesh}(<250 \mu \mathrm{m})$, less than 80 mesh $(<187.5 \mu \mathrm{m})$, and less than 100 mesh $(<150 \mu \mathrm{m})$. Next, $\mathrm{N}_{2}$ adsorption is used to characterize the pore structures of the samples within different particle sizes. Furthermore, to interpret the data, several attributes such as the pore volume, surface area, fractal dimension (from the fractal analysis), and heterogeneity index (from the multifractal analysis), are studied and compared between the samples and particle size intervals to provide us with the effect that particle size could have on the pore structure analysis. The results showed that as the particle size varies, the pore structures of the shale samples could get affected. Based on the comparison of the results, it is recommended that a suitable particle size for the shale pore structure characterization in $\mathrm{N}_{2}$ adsorption experiments should be less than 60 mesh $(<250 \mu \mathrm{m})$.
\end{abstract}

\section{Introduction}

Understanding the pore structures is vital to assess the hydrocarbon bearing capacity of unconventional reservoirs, particularly shale formations. The nanometer to micrometer pores in such reservoirs (e.g., shale reservoirs) could act as the flow path for hydrocarbons, deciding the transportation ability during production [1-8]. Due to this, understanding the pore structures can reveal both pore space and flow abilities of the formation. The gas adsorption method especially $\mathrm{N}_{2}$ gas adsorption, which can measure the pore sizes less than $200 \mathrm{~nm}$, has become one of the most popular methods to characterize the pore structures in shale formations [9-12]. Due to the low permeability nature of shale samples, using larger piece aliquots to perform the $\mathrm{N}_{2}$ adsorption could cause low diffusion of gas molecules to access the pores. Therefore, crushing the samples, to create accessibility for gas molecules to enter the pores in order to achieve the equilibrium in a reasonable time span, is recommended by the Gas Research Institute [13-16].

Although the above general guide line, the appropriate range of particle size for the gas adsorption experiment, $\mathrm{N}_{2}$ adsorption in this study, is still a topic up for argument, and different particle size recommendations have been given, Kulia and Prassad [17] found that the particle size for gas adsorption should be less than $425 \mu \mathrm{m}$ (40 mesh). Other studies have found that using a 60 mesh sieve for crushed particles would be more suitable [18-21]. Tian et al. [22] and Shao et al. [23] suggested using a particle size between 178 and $250 \mu \mathrm{m}$ (60-80 mesh) to study the pore structures. 
For determining the accurate particle size necessary for gas adsorption, Chen et al. [24] compared the pore structures of samples with varying particle sizes: 4 mesh $(<4760 \mu \mathrm{m})$, 20 mesh $(<841 \mu \mathrm{m})$, and 60 mesh $(<250 \mu \mathrm{m})$. Although a good range of particle sizes were studied, they did not investigate the impact of finer particle sizes on pore structures (e.g., $<150 \mu \mathrm{m}$ (100 mesh)).

Mastalera et al. [25] compared the pore structures by assigning different particle sizes: from chunks to the particle size less than $0.063 \mathrm{~mm}$ (230 mesh). Hazra et al. [26] divided the particle sizes into five different groups and studied their pore structures in a larger range of sizes, ranging from $<1 \mathrm{~mm}$ (18 mesh) to $<53 \mu \mathrm{m}$ (270 mesh). In their study, they regarded the particle size ranging from $212 \mu \mathrm{m}$ (70 mesh) to $75 \mu \mathrm{m}$ (200 mesh) as one group and compared the pore structures with the pore structures from other particle size groups (i.e., groups with particle size from $1 \mathrm{~mm}$ to $500 \mu \mathrm{m}$, $212-75 \mu \mathrm{m}$, and $75-53 \mu \mathrm{m}$, respectively). Mastalerz et al. [25] studied the effect of the particle size on the equilibrium time and equilibration pressure and found that the sample with large particle size needs more time to equilibrate. However, if the particle size is too fine, more pores could be generated; the nanopore information could be distorted [26]. Considering studies by Hazra et al. [26] and Mastalera et al.[25], the particle size intervals that were analyzed was 70-200 mesh and 60-200 mesh which is too wide. Hence, the effect of shorter ranges of particle size (e.g., 40-100 mesh) on pore structures is still ambiguous and requires further investigations.

Based on what was said above, in this study, five shale samples were collected, and the effect of smaller particle size on their pore structures is analyzed. Shale samples were crushed in different particle size categories (40 mesh, 60 mesh, 80 mesh, and 100 mesh, respectively), and their pore structures were examined by $\mathrm{N}_{2}$ adsorption to reveal how the size of particles would impact the pore structure results and their interpretation.

\section{Samples and Methods}

2.1. Sample Preparation. Five shale samples were collected, and first were characterized for their organic matter content and mineralogy. The mineral composition shows that clay and quartz are the two major components in these shale samples where the clay mineral content ranges from $17.5 \mathrm{wt} . \%$ (sample 4) to $48.0 \mathrm{wt} \%$ (sample 2), and the quartz content varies from $32.4 \mathrm{wt} . \%$ (sample 2) to $54.4 \mathrm{wt} . \%$ (sample 3). Feldspar, dolomite, and pyrite with smaller percentages also exist in these shale samples. The total organic content of these samples was measured from $13.21 \mathrm{wt} . \%$ (sample 4) to 7.50 wt.\% (sample 5), explaining that these shale samples are rich in organic matter. The $T_{\max }$ of sample 1-sample 5 was $435,430,433,437$, and $411^{\circ} \mathrm{C}$, respectively, indicating that sample 2 and sample 5 are thermally immature while the rest are in the early oil window stage.

2.2. Nitrogen Gas Adsorption Analysis. Next, samples were homogenized into 4 separate pieces, and then, each piece was further crushed into different mesh sieves (40 mesh for part 1, 60 mesh for part 2, 80 mesh for part 3, and 100 mesh for part 4, respectively). Homogenization is the process of breaking several pieces from a single intact sample for different studies in order to limit the bias of the mineral compositions and reducing the effect of variations that could be in different representative aliquot. Next, around 2 grams from each mesh was further dried at $105^{\circ} \mathrm{C}$ for 8 hours before the $\mathrm{N}_{2}$ adsorption analysis. The instrument that was utilized in this study is the Micromeritics ${ }^{\circledR}$ Tristar II apparatus and the $\mathrm{N}_{2}$ adsorption volume was measured over the relative equilibrium adsorption pressure $\left(P / P_{0}\right)$ ranging from 0.01 to 0.99 .

2.3. Fractal Analysis and Multifractal Analysis. The fractal theory is a powerful tool to quantify the complexity of the pore structures of porous medium including geomaterials. The FHH (Frenkel-Halsey-Hill) model is one of the most mainstream fractal models that has been commonly used for shale rock studies [27] and is expressed as:

$$
\ln V=C+(D-3) \ln \left(\ln \left(\frac{1}{p / p_{0}}\right)\right.
$$

where $V$ is the total volume of the adsorption, $P$ is the equilibrium pressure, $P_{0}$ is the saturated vapour pressure of the adsorption, and $D$ is the fractal dimension. $C$ is the constant which can be derived from curve fitting. Once the adsorption data (relative pressure $P / P_{0}$ and the adsorption quantity) is quantified, through Equation (1), the fractal dimension from the slope of the linear part of the curves can be obtained.

It is important to note that the multifractal method which is an extension of the fractal analysis usually uses the box counting method for dimensional analysis. In this method, a set of boxes with equal length $(\varepsilon)$ is used to cover the pore size distribution curve. Then, through density functional theory, a power law relationship as follows is defined ([28, 29]):

$$
P_{i}(\varepsilon) \sim \varepsilon^{\alpha_{i}},
$$

where $I$ is the index for each box laid over the set for the $\varepsilon$ and $P_{i}(\varepsilon)$ is the probability of mass at $I$ relative to the total mass for a box size. Finally, $N_{i}(\varepsilon)$ is the number of mass in any box with the total mass in all boxes for this $\varepsilon$ as below:

$$
\begin{aligned}
N_{T} & =\sum_{i=1}^{N_{\varepsilon}} N_{i}(\varepsilon), \\
P_{i}(\varepsilon) & =\frac{N_{i}(\varepsilon)}{N_{T}}-.
\end{aligned}
$$

$q$ is defined as an arbitrary range of values (in this study, $q$ is from -10 to 10 ) to use as exponents for distorting the data set. For the box size $\varepsilon$, the sum of all mass probabilities distorted can be calculated as [30]:

$$
I_{q(\varepsilon)}=\sum_{i=1}^{N_{\varepsilon}} P_{i}(\varepsilon)^{q}
$$



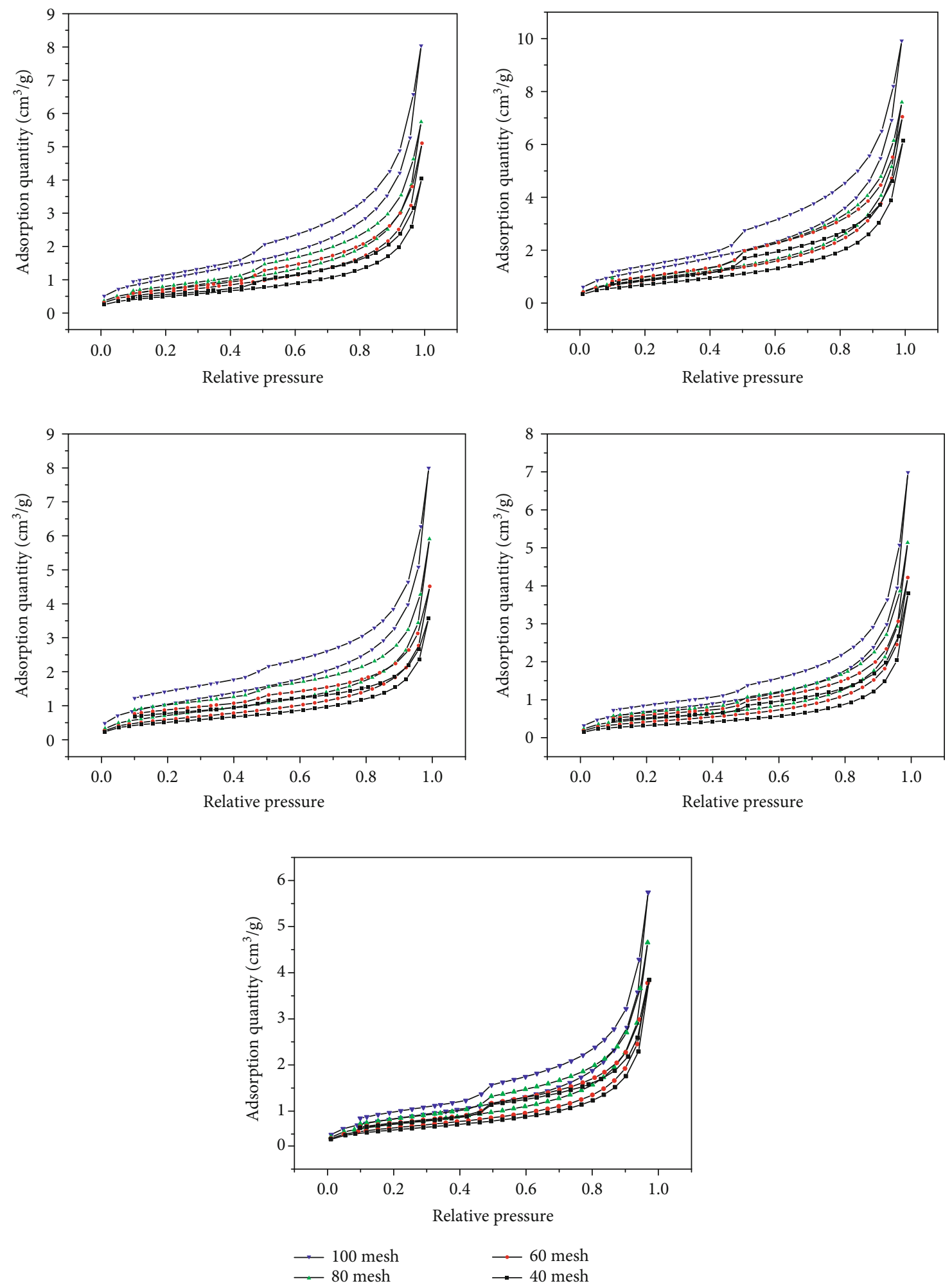

FIgURE 1: Adsorption isotherms of shale samples with different particle sizes. 


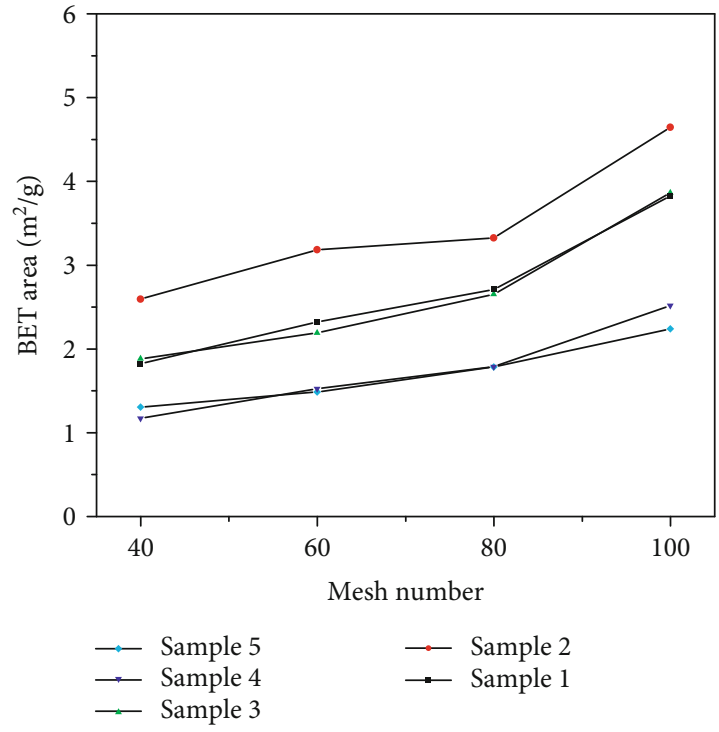

(a) BET surface area
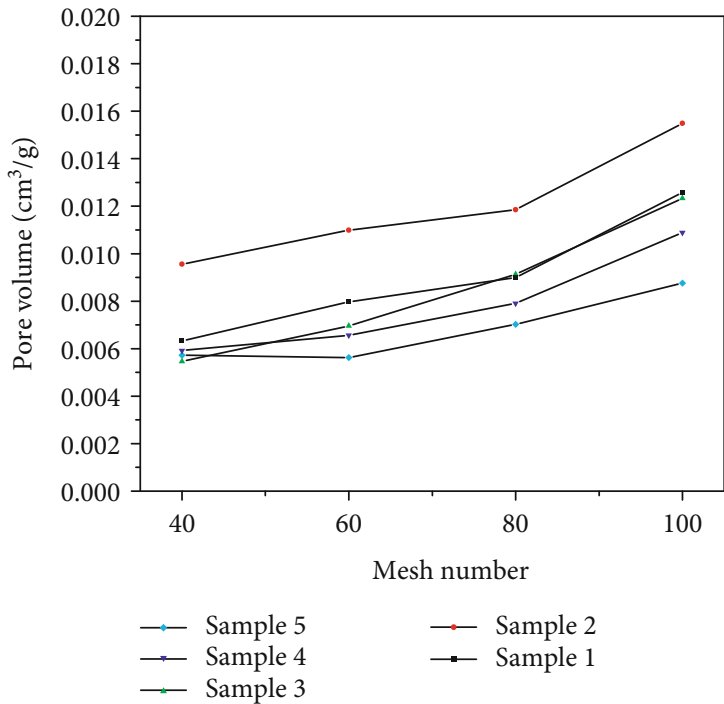

(b) Pore volume

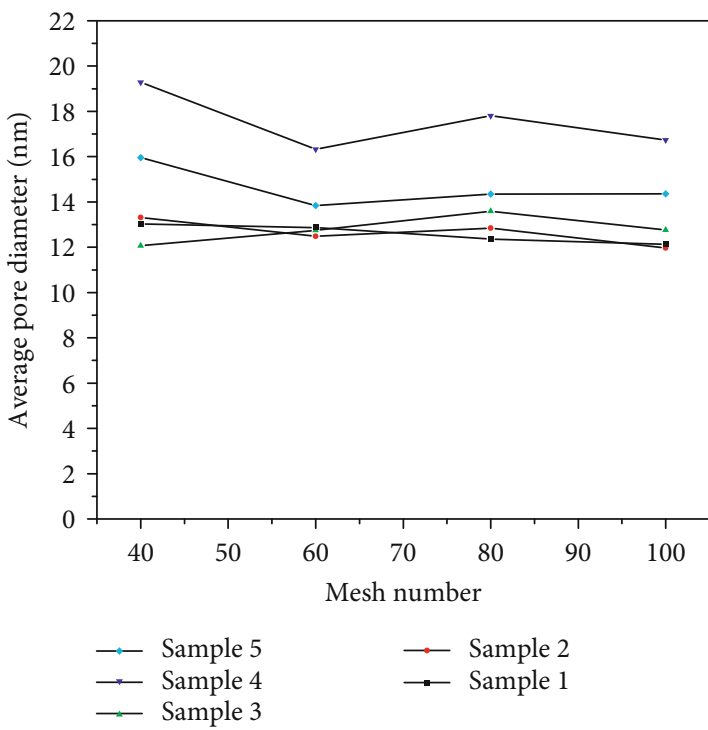

(c) Average pore size

FIgURE 2: The effect of the particle size on the pore parameters.

For the box size $\varepsilon$, the magnitude of distorted mass probability at a specific box compared to the distorted sum of all boxes can be quantified as:

$$
u_{i}(q, \varepsilon)=\frac{P_{i}(\varepsilon)^{q}}{I_{q(\varepsilon)}}
$$

where $I_{q(\varepsilon)}$ can be expressed as [31]:

$$
I_{q(\varepsilon)} \sim \mathcal{\varepsilon}^{\tau_{(q)}} .
$$

Then, $\tau_{q}$ can be calculated as:

$$
\tau_{q}=\lim _{\varepsilon \rightarrow 0}\left(\frac{\log I_{q(\varepsilon)}}{\log \varepsilon}\right) .
$$

$$
D_{q} \text { will be: }
$$

$$
D_{q}=\frac{\tau_{q}}{q-1}=\frac{\lim _{\varepsilon \rightarrow 0}\left(\left(\log I_{q(\varepsilon)}\right) /(\log \varepsilon)\right)}{q-1} .
$$

If $q=1$,

$$
D_{1}=\lim _{\varepsilon \rightarrow 0} \frac{\sum_{i=1}^{N(\varepsilon)} p_{i}(\varepsilon) \ln p_{i}(\varepsilon)}{\ln (\varepsilon)} .
$$

\section{Results and Discussions}

3.1. The Adsorption Isotherms of the Shale Samples with Different Particle Sizes. The adsorption isotherms that are obtained from the shale samples crushed into different 

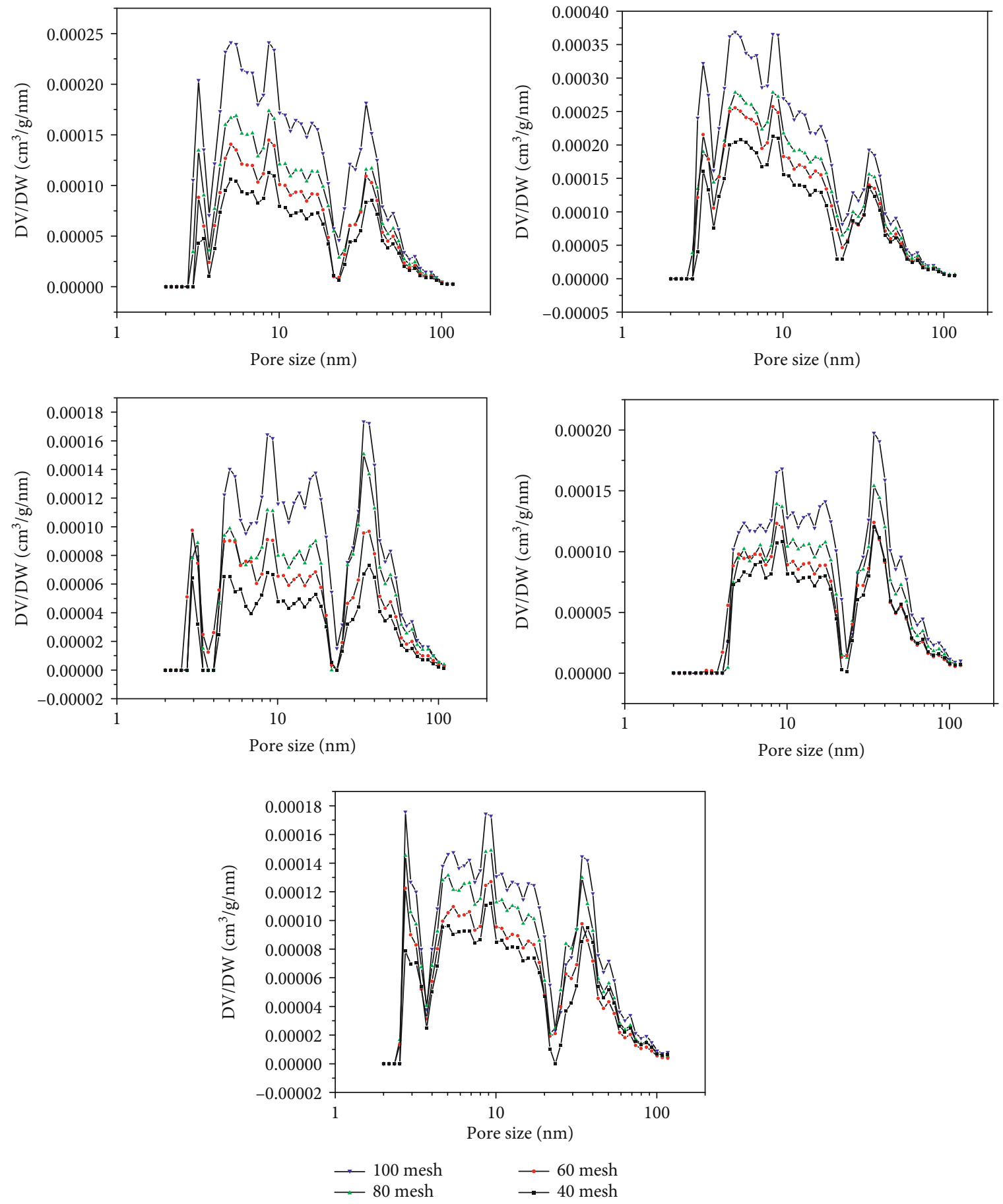

FIgUre 3: The pore size distribution (PSD) of the samples with different particle sizes.

particle sizes can be seen in Figure 1. Although these shale samples are at different thermal maturity levels, the adsorption isotherms still shows some similarities considering their difference in particle size. For instance, in samples with smaller particle size, under the same relative pressure, the adsorption quantity is recorded higher. This indicates that the adsorption capacity of the sample has increased. Considering sample 1 as an example, as the particle size decreased from 40 mesh $(<375 \mu \mathrm{m})$ to 100 mesh $(<150 \mu \mathrm{m})$, the adsorption volume has increased from $4.04 \mathrm{~cm}^{3} / \mathrm{g}$ to
$8.04 \mathrm{~cm}^{3} / \mathrm{g}$. Based on the recommendations by the International Union of Pure and Applied Chemistry (IUPAC), the shape of the adsorption isotherms of the samples with different particle sizes all belong to type IV, showing the existence of mesopores $(2-50 \mathrm{~nm})$ and macropores $(>50 \mathrm{~nm})$ [32]. Moreover, hysteresis loop is observed between the adsorption and the desorption isotherm branch in all samples. The shape of the hysteresis loop points out that there is an abundance of slit-shape pores in these shale samples although being different in particle size [32]. Ultimately, as the particle size has 


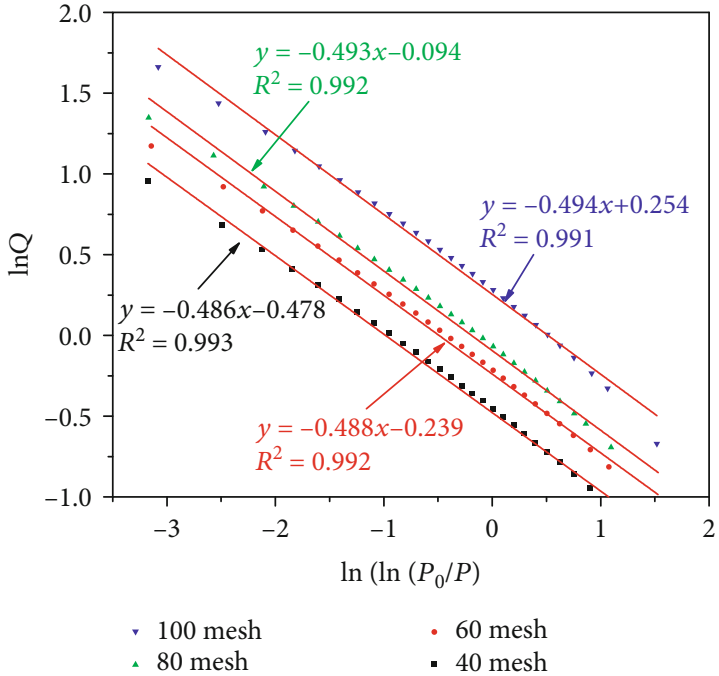

Figure 4: The effect of the particle size on the fractal dimensions of the shale sample (sample 1).

changed, the adsorption isotherms are somehow affected which can be regarded as alteration in sample microstructure.

\subsection{Comparison of the Pore Structures of the Sample with} Different Particle Sizes. The surface area is calculated from the Brunauer-Emmett-Teller (BET) method [33], and the pore volume of the shale samples is derived from the $\mathrm{BJH}$ (Barrett, Joyner, and Halenda) method [34], which is based on the Kelvin equation. Figure 2(a) explains, as the particle size decreases (the mesh number increases), regardless of the sample, the BET-calculated surface area increases. In all samples, an abrupt change in the BET-calculated surface area when the particle size is decreased from $<187.5 \mu \mathrm{m}$ ( 80 mesh) to $<150 \mu \mathrm{m}$ (100 mesh) is found. This could be due to either creation of new surfaces or connection of isolated pores. The BET surface area of the sample in the 100 mesh interval is around twice the BET surface area of the sample in the 40 mesh interval. In addition, the BET surface area of sample 1 has increased from $1.82 \mathrm{~m}^{2} / \mathrm{g}$ (40 mesh) to $3.83 \mathrm{~m}^{2} / \mathrm{g}(100$ mesh). Similarly, the pore volume also has increased as the particle size is decreased (Figure 2(b)). Considering sample 1 as an example, the pore volume in the 100 mesh particle size is $0.0074 \mathrm{~cm}^{3} / \mathrm{g}$ which is twice more than the pore volume in the 100 mesh particle size $\left(0.0036 \mathrm{~cm}^{3} / \mathrm{g}\right)$. Furthermore, the sample that is crushed to 40 mesh size was measured with the smallest BET surface area and pore volume. Figure 2(c) exhibits the average pore size of the samples based on their particle sizes. It is deduced as the particle size decreases from $375 \mu \mathrm{m}$ ( 40 mesh) to $250 \mu \mathrm{m}$ (60 mesh), the average pore size shows an apparent change. On the other hand, further change in particle size beyond $250 \mu \mathrm{m}(60$ mesh) to 100 mesh, the average pore size does not show a meaningful trend, although fluctuations are observed.

The pore size distribution (PSD) curves of samples with different particle sizes are presented in Figure 3 where a multimodal distribution is seen in all samples. Although there are discrepancies in thermal maturity, these samples still have

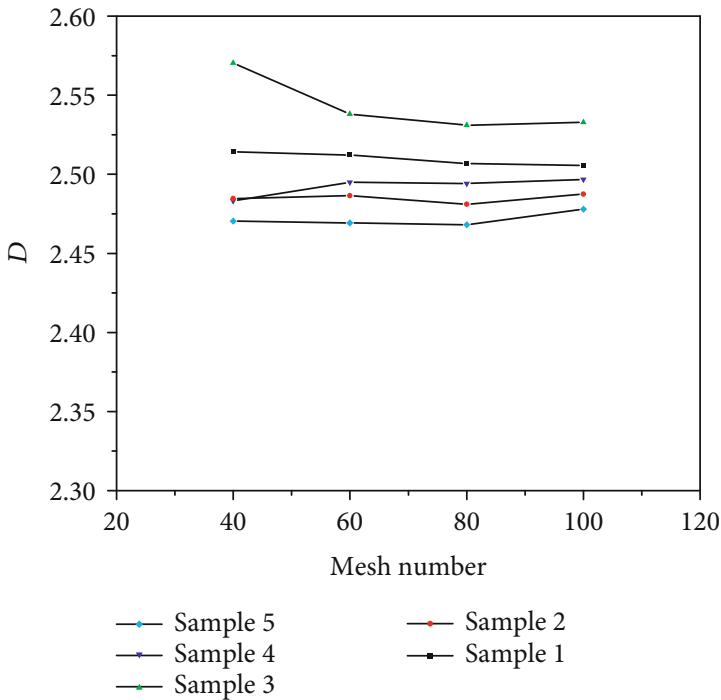

FIgURE 5: Summary of the fractal values of the samples with different particle sizes.

similar peaks at certain pore sizes (e.g., the peak with the pore size between 30 and $40 \mathrm{~nm}$ ). The importance of thermal maturity is due to the fact that at higher maturities, organic matter should have developed more organic pores and it is expected that change to be revealed in adsorption tests. A closer look explains that samples 1,2,3, and 5 have a discernable peak around $3 \mathrm{~nm}$ which is absent in sample 4 . Liu et al. [35] applied the Gaussian distribution to distinguish the presence of various pore groups in shale samples based on the peaks of different pore sizes in a PSD curve. Considering their conclusions, Liu et al. [35], we further deconvoluted different possible pore groups in the PSD curve. On the one hand, it is found that as the particle size decreases, the number of pores in each group has increased following the Liu et al. [35] method. For example, in sample 1, highest frequencies of PSD of the 40 mesh particles are around 3, 5, 9, 17, and $34 \mathrm{~nm}$, respectively, but staying the same in smaller particle sizes or higher meshes.

\subsection{Comparison of the Fractal Dimensions of Samples with Different Particle Sizes}

3.3.1. Fractal Dimension. Based on the results by the FHH model, we calculated the fractal dimensions of all the samples based on different particle sizes. Figure 4 displays an example of the fractal analysis of sample 1 with different particle sizes. The plots illustrates that a linear correlation exists between the $\ln Q$ and $\ln \left(\ln \left(P_{0} / P\right)\right)$, indicating that the pore size distributions of sample 1 with different particle sizes all follow the fractal behavior. The summary of the fractal dimensions of all samples while considering variations in particle sizes can be seen in Figure 5. The fractal dimension values are all located in a reasonable range of fractal values (between 2 and 3) $[36,37]$. However, comparing different samples, the effect that the particle size has on the fractal dimension results is not similar. In this regard, it is observed as the particle size decreases, the fractal dimension of sample 1, 2, 4, 


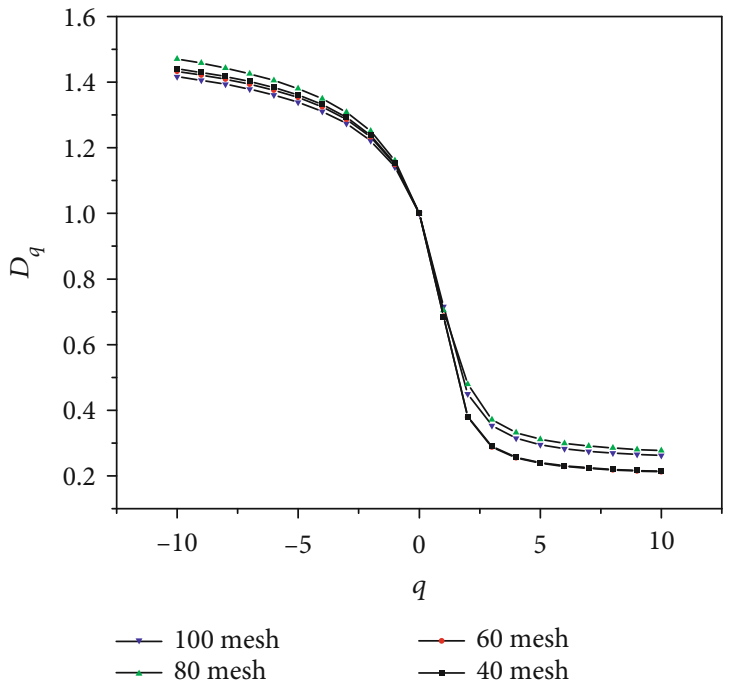

Figure 6: The correlation between $D_{q}$ and q of sample 1 .

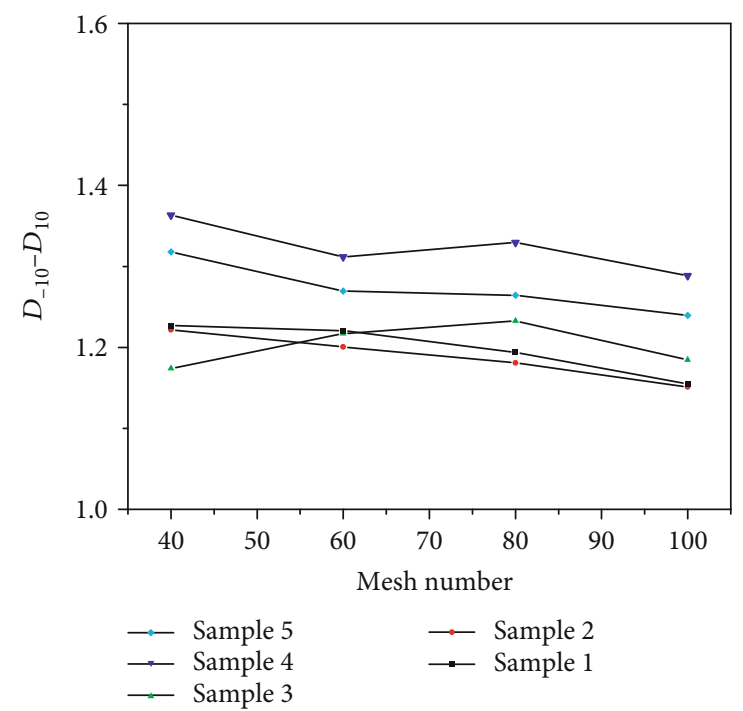

Figure 7: The effect of the particle size on the heterogeneity of the pore size distribution.

and 5 does not change significantly (Figure 4), whereas, in sample3, as the particle size decreases from $<375 \mu \mathrm{m}(40$ mesh) to $<250 \mu \mathrm{m}$ (60 mesh), the fractal dimension demonstrates an evident decrease. On the contrary, as the particle size continues to decrease from $<250 \mu \mathrm{m}$ (60 mesh) to $<150 \mu \mathrm{m}$ (100 mesh), the fractal dimension does not change notably. Additionally, in a particle size range of less than $250 \mu \mathrm{m}(60$ mesh), the fractal dimensions comparing five samples follow the order: sample $3>$ sample $1>$ sample $4>$ sample $2>$ sample 5. This means as the particle size decreases, up to $<150 \mu \mathrm{m}$, the order of fractal dimension change among these five samples remains the same (sample 3 has the largest fractal dimension and sample 5 the smallest value).

3.3.2. Multifractal Dimension. Based on Section 2.3, we calculated the generalized dimensions $D_{q}$ from $\mathrm{N}_{2}$ adsorption of all the shale samples with different particle sizes. Here, in
$D_{q}$ values (in this study, $q$ is from -10 to 10 ), $D_{0}$ represents the singularity of nonempty boxes containing some value of porosity under successive finer partitions. $D_{1}$ can characterize the concentration degree of the pore size distribution along the pore size intervals. $D_{2}$ accounts for the behavior of the second sampling moments [31]. The $D_{q}$ value of sample 1 with different particle sizes can be seen in Figure 6 where $D_{0}>D_{1}>D_{2}$ order of change exists in sample 1 . This is indicating that the pore size distribution of sample 1, considering the existence of different particle sizes, presents the multifractal behavior [31]. The difference between the $D_{-10}$ and $D_{10}$ can be used to quantify the heterogeneity of the pore size distribution of the sample [38]. Based on this, the larger $D_{-10}-D_{10}$ value corresponds to a higher degree of heterogeneity in the pore size distribution. The $D_{-10}-D_{10}$ values of all studied samples with different particle sizes are calculated, and the results can be seen in Figure 7. Based on the results, as the particle size decreases, the heterogeneity index $\left(D_{-10}-D_{10}\right)$ fluctuates with a slight decreasing trend. This infers that the change in particle size has some impact on the heterogeneity that is present in the pore structures. Comparing all samples, the heterogeneity index when particle size is $<250 \mu \mathrm{m}$ ( $60 \mathrm{mesh})$ is very close to the heterogeneity index when the particle size is $<187.5 \mu \mathrm{m}$ ( $80 \mathrm{mesh}$ ). Consequently, as the particle size further decreases to $<150 \mu \mathrm{m}$ (100 mesh), the heterogeneity index decreases, regardless of the sample number. In sample 1 as an example, the heterogeneity index decreases from 1.19 to 1.15 as the particle size is reduced from $<187.5 \mu \mathrm{m}$ ( $80 \mathrm{mesh})$ to $<150 \mu \mathrm{m}$ (100 mesh).

\section{Conclusions}

In this study, it was shown that crushing the samples to a smaller size could change how the pore structures of shale samples are interpreted. This was overall inferred due to creation of fractures, pore collapse, or generation of new pores and surface areas in the samples from the crushing process. The adsorption isotherms displayed that, as the particle size decreases, the adsorption capacity increases. In addition, the surface area and pore volume also increased. The BET surface area of the sample in the 100 mesh particle size can be twice the value of the sample in the 40 mesh range. The average pore size varied clearly when the particle sizes decreased from 40 mesh to 60 mesh. However, as the particle size decreased further to 100 mesh, the average pore size does not change significantly. Next, the pore size distribution curves of samples showed similar peak locations, indicating that crushing the sample from 40 mesh to 100 mesh does not introduce new pores.

Ultimately, fractal analysis defined as the particle size decreases from 40 mesh to 60 mesh; the fractal dimension would vary (the reduction of the fraction dimension for sample 3 and the increase of the fractal dimension for sample 4). However, the fractal dimension remains almost constant as the particle size becomes finer from 60 mesh to 100 mesh. In this study, the surface area, pore volume, the average pore size, and the fractal dimension of the samples in the 40 mesh range would differ compared to other particle size range. In 
this regard, we conclude, if the particle size is too large, the adsorption result might not be reliable due to incomplete equilibration and slow gas diffusion. Ultimately, the multifractal dimension result exhibited that, as the particle size decreases from 80 mesh to 100 mesh, the heterogeneity index of the shale samples decreases, meaning the samples are becoming more homogeneous. Overall, from this study, considering the pore structure parameters (pore surface area, pore volume, average pore radius, pore complexity, and heterogeneity), it is recommended that a reasonable particle size for should be either 60 mesh $(<250 \mu \mathrm{m})$ or 80 mesh $(<187.5 \mu \mathrm{m})$, and $60 \mathrm{mesh}$ is considered as the reasonable size since finer scale requires additional effort.

\section{Data Availability}

The data used to support the findings of this study are available from the corresponding author upon request.

\section{Conflicts of Interest}

The authors declare that they have no conflicts of interest.

\section{Acknowledgments}

The authors would appreciate anonymous reviewers who refereed this paper for all their valuable comments that helped to greatly improve the quality of the paper.

\section{References}

[1] R. M. Bustin, A. M. M. Bustin, A. Cui, D. Ross, and V. M. Pathi, "Impact of shale properties on pore structure and storage characteristics," in In SPE shale gas production conference, Society of Petroleum Engineers, 119892-MS 1, pp. 1-28, 2008.

[2] B. Liu, Y. Song, K. Zhu, P. Su, X. Ye, and W. Zhao, "Mineralogy and element geochemistry of salinized lacustrine organic-rich shale in the middle Permian Santanghu Basin: implications for paleoenvironment, provenance, tectonic setting and shale oil potential," Marine and Petroleum Geology, vol. 120, 2020.

[3] B. Liu, Y. Yang, J. Li, Y. Chi, J. Li, and X. Fu, "Stress sensitivity of tight reservoirs and its effect on oil saturation: a case study of Lower Cretaceous tight clastic reservoirs in the Hailar Basin, Northeast China," Journal of Petroleum Science and Engineering, vol. 184, 2020.

[4] K. Liu and M. Ostadhassan, "The impact of pore size distribution data presentation format on pore structure interpretation of shales," Advances in Geo-Energy Research, vol. 3, no. 2, pp. 187-197, 2019.

[5] R. G. Loucks, R. M. Reed, S. C. Ruppel, and U. Hammes, "Spectrum of pore types and networks in mudrocks and a descriptive classification for matrix-related mudrock pores," AAPG Bulletin, vol. 96, no. 6, pp. 1071-1098, 2012.

[6] C. Lyu, Y. Zhang, C. Li et al., "Pore characterization of Upper Ordovician Wufeng Formation and Lower Silurian Longmaxi Formation shale gas reservoirs, Sichuan Basin, China," Journal of Natural Gas Geoscience, vol. 5, no. 6, pp. 327-340, 2020.

[7] X. Wang, J. Hou, S. Song et al., "Combining pressurecontrolled porosimetry and rate-controlled porosimetry to investigate the fractal characteristics of full-range pores in tight oil reservoirs," Journal of Petroleum Science and Engineering, vol. 171, pp. 353-361, 2018.

[8] X. Wang, J. Hou, S. Li et al., "Insight into the nanoscale pore structure of organic-rich shales in the Bakken Formation, USA," Journal of Petroleum Science and Engineering, vol. 191, p. 107182, 2020.

[9] G. R. Chalmers, R. M. Bustin, and I. M. Power, "Characterization of gas shale pore systems by porosimetry, pycnometry, surface area, and field emission scanning electron microscopy/transmission electron microscopy image analyses: examples from the Barnett, Woodford, Haynesville, Marcellus, and Doig units," AAPG Bulletin, vol. 96, no. 6, pp. 10991119, 2012.

[10] C. R. Clarkson, N. Solano, R. M. Bustin et al., "Pore structure characterization of North American shale gas reservoirs using USANS/SANS, gas adsorption, and mercury intrusion," Fuel, vol. 103, pp. 606-616, 2013.

[11] K. Liu, M. Ostadhassan, L. Sun et al., "A comprehensive pore structure study of the Bakken Shale with SANS, $\mathrm{N}_{2}$ adsorption and mercury intrusion," Fuel, vol. 245, pp. 274-285, 2019.

[12] M. Wei, L. Zhang, Y. Xiong, J. Li, and P.'a. Peng, "Nanopore structure characterization for organic-rich shale using the non-local-density functional theory by a combination of $\mathrm{N}_{2}$ and $\mathrm{CO}_{2}$ adsorption," Microporous and Mesoporous Materials, vol. 227, pp. 88-94, 2016.

[13] X. Li, Z. Gao, S. Fang, C. Ren, K. Yang, and F. Wang, "Fractal characterization of nanopore structure in shale, tight sandstone and mudstone from the Ordos Basin of China using nitrogen adsorption," Energies, vol. 12, no. 4, pp. 583-600, 2019.

[14] Y. Liu, B. Zhang, Y. Dong, Z. Qu, and J. Hou, "The determination of variogram in the presence of horizontal wells - an application to a conglomerate reservoir modeling, East China," Journal of Petroleum Science and Engineering, vol. 173, pp. 512-524, 2019.

[15] D. L. Luffel and F. K. Guidry, "New core analysis methods for measuring reservoir rock properties of Devonian shale," Journal of Petroleum Technology, vol. 44, no. 11, pp. 1184-1190, 1992.

[16] R. Yang, A. Jia, Q. Hu, X. Guo, and M. Sun, "Particle size effect on water vapor sorption measurement of organic shale: one example from Dongyuemiao Member of Lower Jurassic Ziliujing Formation in Jiannan Area of China," Advances in GeoEnergy Research, vol. 4, no. 2, pp. 207-218, 2020.

[17] U. Kuila and M. Prasad, "Application of nitrogen gasadsorption technique for characterization of pore structure of mudrocks," The Leading Edge, vol. 32, no. 12, pp. 1478-1485, 2013.

[18] M. M. Labani, R. Rezaee, A. Saeedi, and A. AlHinai, "Evaluation of pore size spectrum of gas shale reservoirs using low pressure nitrogen adsorption, gas expansion and mercury porosimetry: a case study from the Perth and Canning Basins, Western Australia," Journal of Petroleum Science and Engineering, vol. 112, pp. 7-16, 2013.

[19] Y. Liu, K. Ma, J. Hou, L. Yan, and F. Chen, "Diagenetic controls on the quality of the Middle Permian Lucaogou Formation tight reservoir, southeastern Junggar Basin, northwestern China," Journal of Asian Earth Sciences, vol. 178, pp. 139-155, 2019.

[20] M. Mastalerz, L. He, Y. B. Melnichenko, and J. A. Rupp, "Porosity of coal and shale: insights from gas adsorption and 
SANS/USANS techniques," Energy \& Fuels, vol. 26, no. 8, pp. 5109-5120, 2012.

[21] D. J. K. Ross and R. M. Bustin, "The importance of shale composition and pore structure upon gas storage potential of shale gas reservoirs," Marine and Petroleum Geology, vol. 26, no. 6, pp. 916-927, 2009.

[22] H. Tian, L. Pan, X. Xiao, R. W. T. Wilkins, Z. Meng, and B. Huang, "A preliminary study on the pore characterization of Lower Silurian black shales in the Chuandong Thrust Fold Belt, southwestern China using low pressure $\mathrm{N}_{2}$ adsorption and FE-SEM methods," Marine and Petroleum Geology, vol. 48, pp. 8-19, 2013.

[23] X. Shao, X. Pang, Q. Li et al., "Pore structure and fractal characteristics of organic-rich shales: a case study of the lower Silurian Longmaxi shales in the Sichuan Basin, SW China," Marine and Petroleum Geology, vol. 80, pp. 192-202, 2017.

[24] Y. Chen, L. Wei, M. Mastalerz, and A. Schimmelmann, "The effect of analytical particle size on gas adsorption porosimetry of shale," International Journal of Coal Geology, vol. 138, no. 1, pp. 103-112, 2015.

[25] M. Mastalerz, L. Hampton, A. Drobniak, and H. Loope, "Significance of analytical particle size in low-pressure N2 and $\mathrm{CO} 2$ adsorption of coal and shale," International Journal of Coal Geology, vol. 178, pp. 122-131, 2017.

[26] B. Hazra, D. A. Wood, V. Vishal, and A. K. Singh, "Pore characteristics of distinct thermally mature shales: influence of particle size on low-pressure $\mathrm{CO} 2$ and $\mathrm{N} 2$ adsorption," Energy \& Fuels, vol. 32, no. 8, pp. 8175-8186, 2018.

[27] P. Pfeifer and M. W. Cole, "Fractals in surface science: scattering and thermodynamics of adsorbed films," New Journal of Chemistry, vol. 14, no. 3, pp. 221-232, 1990.

[28] B. B. Chaudhuri and N. Sarkar, "Texture segmentation using fractal dimension," IEEE Transactions on Pattern Analysis and Machine Intelligence, vol. 17, no. 1, pp. 72-77, 1995.

[29] D. A. Russell, J. D. Hanson, and E. Ott, "Dimension of strange attractors," Physical Review Letters, vol. 45, no. 14, pp. 1175$1178,1980$.

[30] A. N. D. Posadas, D. Giménez, M. Bittelli, C. M. P. Vaz, and M. Flury, "Multifractal characterization of soil particle-size distributions," Soil Science Society of America Journal, vol. 65, no. 5, pp. 1361-1367, 2001.

[31] J. P. Ferreiro, M. Wilson, and E. V. Vázquez, "Multifractal description of nitrogen adsorption isotherms," Vadose Zone Journal, vol. 8, no. 1, pp. 209-219, 2009.

[32] M. Thommes, K. Kaneko, A. V. Neimark et al., "Physisorption of gases, with special reference to the evaluation of surface area and pore size distribution (IUPAC Technical Report)," Pure and Applied Chemistry, vol. 38, no. 1, p. 25, 2011.

[33] S. Brunauer, P. H. Emmett, and E. Teller, "Adsorption of gases in multimolecular layers," American chemical journal, vol. 60, no. 2, pp. 309-319, 1938.

[34] E. P. Barrett, L. G. Joyner, and P. P. Halenda, "The determination of pore volume and area distributions in porous substances. I. Computations from nitrogen isotherms," Journal of the American Chemical Society, vol. 73, no. 1, pp. 373-380, 1951.

[35] K. Liu, M. Ostadhassan, J. Zhou, T. Gentzis, and R. Rezaee, "Nanoscale pore structure characterization of the Bakken shale in the USA," Fuel, vol. 209, pp. 567-578, 2017.

[36] F. Wang and S. Li, "Determination of the surface fractal dimension for porous media by capillary condensation,"
Industrial \& Engineering Chemistry Research, vol. 36, no. 5, pp. 1598-1602, 1997.

[37] X. Wang, Y. Liu, J. Hou et al., "The relationship between synsedimentary fault activity and reservoir quality-a case study of the Ek1 formation in the Wang Guantun area, China," Interpretation, vol. 8, no. 3, pp. sm15-sm24, 2020.

[38] K. Liu, M. Ostadhassan, J. Zou et al., "Multifractal analysis of gas adsorption isotherms for pore structure characterization of the Bakken Shale," Fuel, vol. 219, pp. 296-311, 2018. 